\title{
并联式下肢康复外骨骼运动学及工作空间分析
}

\author{
陈伟海 徐颖俊 王建华 张建斌 \\ (北京航空航天大学智能机器人与测控技术实验室 北京 100191)
}

\begin{abstract}
摘要: 针对下肢痽疾病人, 设计一种新型的平面三自由度并联式下肢外骨骼机构进行研究, 运用指数积公式对该机构的位置 正反解进行研究分析, 同时对该机构的雅可比矩阵和运动奇异性进行分析。以工作空间最大为目标函数, 分析影响并联机构 工作空间的主要因素, 如连杆长度、运动副转角、人体关节角度等对工作空间的约束。采用边界搜索法确定工作空间边界, 计算出相应形状的工作空间面积, 分析各机构参数对工作空间的影响。在运动学分析的基础上, 以满足人体正常行走时鯑关 节和膝关节运动范围要求, 以提高机构运动学性能为目标, 进行结构参数优化设计。初步分析结果表明, 对机构参数进行优 化, 能提高运动学性能指标, 该机构能够用于下肢痽梹病人进行康复训练, 为进一步并联机构的设计研究奠定了基础。
\end{abstract}

关键词: 下肢外骨骼；指数积公式；位置正反解；工作空间

中图分类号: TH112

\section{Kinematics and Workspace Analysis of Parallel Lower Limb Rehabilitation Exoskeleton}

\author{
CHEN Weihai XU Yingjun WANG Jianhua ZHANG Jianbin \\ (Laboratory of Intelligent Robot \& Measurement and Control Technology, Beihang University, Beijing 100191)
}

\begin{abstract}
In view of the lower paralysis patients, a new type of 3-DOF parallel lower limb exoskeleton mechanism is designed as the foundation for research, the forward and inverse position solution of the mechanism are analyzed with product of exponentials (POE) formulation, in the meanwhile, the Jacobian matrix and motion singularity of mechanism are also analyzed. Taking the maximum workspace as the objective function, the chief factors influencing the workspace of the parallel mechanism such as the workspace restriction of the length of linkage, the angle of motion pair and the human body's joint angle have been taken into account. The boundary of workspace is determined with the help of boundary search method, and the area of workspace is calculated. The influence of various parameters of the mechanism on the workspace is analyzed. On the basis of kinematics analysis, in order to satisfy the demand of hip's angle and knee's angle range of motion of normal human walking, to improve the mechanism kinematic performance as the goal, optimize the value of structural parameters. Preliminary analysis shows that the kinematical performance can been improved with optimizing the value of the structural parameters. The mechanism can be used to lower limb paralysis patients in rehabilitation training. The study paves the way for the design and analysis of the parallel mechanisms.
\end{abstract}

Key words: lower limb exoskeleton; products of exponentials; forward and inverse position solution; workspace

\section{0 前言}

并联机构具有刚度大, 承载能力高, 输出精度 高等优点 ${ }^{[1]}$ 。近年来在很多领域得到了很大的发展 和应用。外骨骼机器人是一种可穿戴式机器人，用 以增强人的体能, 实现瘫疾病人的康复训练以及辅 助人进行各种操作等。瑞士和苏黎世联邦理工大学

* 国家科技支撑计划资助项目(2012BAI33B00)。20150202 收到初稿, 20150526 收到修改稿
合作研发的 Lokomat 康复训练机器人是目前最为成 熟并已经商业化的康复机器人 ${ }^{[2]}$ 。该机器人采用直 线电动机驱动, 在膝、髋关节处各有 1 个主动的自 由度来实现在行走平面的运动。该系统可以根据患 者身高进行适应性穿戴调节, 适用范围较广。哥伦 比亚大学 AGRAWAL 教授研制的 ALEX III机器 人 $^{[3]}$, 共有 12 个自由度: 盆骨有 4 个自由度, 每条 腿各有 4 个自由度。对每条腿而言, 髋关节 2 个自 由度(内收/外展, 屈/伸), 膝关节 1 个自由度, 踝关 节 1 个自由度。为减轻下肢外骨骼重量, 设计者将 
所有的驱动电动机都放置在外骨骼的后方, 采用带 轮等实现电动机远端驱动。该机器人目前仅利用健 康人做简单的试验, 尚未应用于患者。Rewalk 是由 以色列一家公司设计的一款用于辅助截瘫患者行走 的独立式外骨骼康复机器人 ${ }^{[4]}$ 。该机器人的髋关节 和膝关节在行走方向各有一个旋转自由度, 由直流 电动机直接驱动。患者通过拐杖保持平衡并辅助行 走, 该机器人可以辅助截㿈患者实现坐下、起立、 行走、爬楼梯等动作。

机构运动学包括位姿的正解, 反解, 速度和加 速度的分析。并联机构的位姿正解比位姿逆解要复 杂, 位姿正解多为非线性方程组。刘慧林等 ${ }^{[5]}$ 运用 吴方法得到了 3-RPR 的封闭形式的解。罗佑新 ${ }^{[6]}$ 运 用粗迭代和精迭代求解 3-RPR 机构的正解, 从而提 高了求解效率。并联机构的奇异性 ${ }^{[7-9]}$ 也是并联机构 目前的主要研究内容之一。并联机构的奇异位形的 问题是由 HUNT 于 1994 年第一次提出来, 随后黄 真和 FICHER 运用数学工具分别提出了 Stewart 平 台的奇异位形, GOSSELIN 等将速度的输入输出关 系表达式 $\boldsymbol{A} x+\boldsymbol{B} y=0$, 然后根据 $\boldsymbol{A}, \boldsymbol{B}$ 矩阵分别等于 零, 或者都等于零, 将奇异位形分为三种, 分别是 位形奇异, 边界奇异和构型奇异。MERLET 和 HAO 等利用线几何空间线从和线汇的结论, 对并联机构 做了不同的研究。ZHATANOV 等对一般的非几余 机构的奇异位形做了研究基于运动学奇异的速度方 程建模。BASU 等由共切概念建立了集合关系, 满 足集合条件其 Jacobian 矩阵为零, 对众多多环空间 机构进行了奇异位形的研究。赵新华等 ${ }^{[10]}$ 根据机构 的约束条件简化了机构的奇异位形条件方程。

机构的工作空间是指机器人末端执行器的工 作区域, 也是判断机构性能的主要指标之一。同串 联机构相比, 并联机构的工作空间较小, 而且其解 析求解是一个非常复杂的过程。GOSSELIN 等 ${ }^{[11]}$ 运用圆弧相交的方法, 分析了 3-RPR 并联机构的工 作空间。高峰等 ${ }^{[7]}$ 建立了多种并联机构的空间模型, 运用相对量纲一机构主要是涉及并联机构的对称 性, 得出工作空间体积的算法, 比较详细的绘制了 可达工作空间的体积性能图谱。肖红军 ${ }^{[12]}$ 运用快速 极坐标搜索方法来研究 3-RPR 平面并联机构的工作 空间。获取大工作空间对于并联机构设计是一个重 要的优化目标, 并联机构的工作空间体积优化具有 参数众多、参数敏感性差异大、参间耦合强等特点, 因此作为一个高位非线性问题, 其寻优算法是一个 设计难点。BOUDREAU 等 ${ }^{[13]}$ 利用遗传算法求解三 自由度平面并联机构和 Stewart 机构的空间最优体 积问题。CHEN 等 ${ }^{[14]}$ 利用遗传算法对定姿态的
Stewart 机构工作空间进行优化，保证工作空间可以 包含给定圆柱区域。马晓丽等 ${ }^{[15]}$ 研究一种新型 3T-1R 四自由度并联机器人机构, 运用正交遗传算 法对机构工作空间进行优化设计。

从关节运动的原理来看, 肌肉群共同作用牵引 骨骼产生运动, 因此采用并联式的外骨骼关节具有 一定的仿生意义。本文通过对并联机构的选型和机 构性能的分析, 为人体下肢康复训练设计了一种新 型的并联外骨骼机构。本文利用基于旋量理论的指 数积方法, 对并联外骨骼机构建立数学模型, 并对 该机构运动学进行分析。同时在给出约束条件的基 础上, 以提高运动学性能为指标, 对其工作空间进 行分析, 并对机构参数进行优化。该机构通过运动 学的研究, 可以使下肢机构按照人体标准的步态进 行运动, 对下肢功能障碍患者进行康复。人体的下 肢运动局限在一定的运动范围内, 通过对机构工作 空间的分析和优化, 可以使下肢机构的工作空间运 动范围更加接近人体下肢运动范围。

\section{1 并联机构模型}

\section{1 机构模型}

本文设计具有三自由度的串并联机构来实现 关节的外骨骼化, 以提高其仿生性和通用性。平面 三自由度串并联机构模型如图 1 所示。该机构具有 强度高, 负载能力较大等特点。

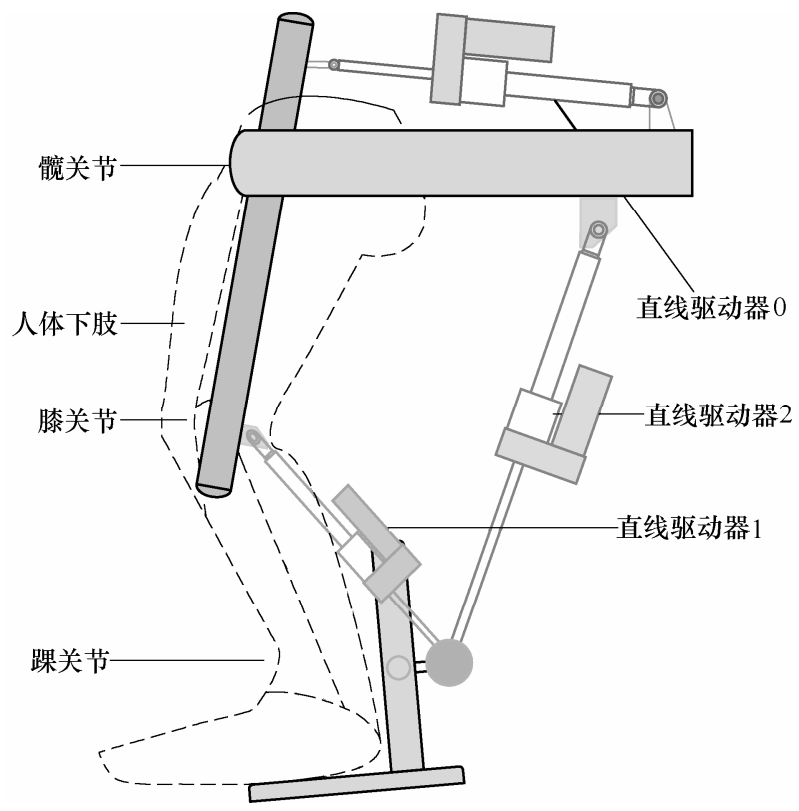

图 1 下肢康复串并联式外骨骼机构模型

下肢外骨骼机构设计主要是包括髋关节设计, 膝关节设计, 踝关节和足底设计, 重点研究的是骼 关节和膝关节设计。 
膝关节由股骨内、外侧踝和胫骨内、外侧踝以 及髌骨组成, 是人体最大且最复杂的关节, 膝关节 的运动并非简单的定轴旋转运动, 而是在旋转运动 上耦合了轴心平移运动。在膝关节处采用两个直线 驱动器来驱动, 驱动器分别由旋转电动机和滚珠丝 杜组成。两个驱动器的上端分别连接在外骨骼大腿 的两个转轴上, 下端同轴连接在下肢外骨骼小腿后 部, 从而构成了一个以膝关节为中心的并联仿生机 构。髋关节可以简化为一个铰链关节。在外骨骼髋 关节处, 采用直线驱动器, 滚珠丝杆的直线运动转 化为外骨骼大腿绕髋关节的转动。

这样外骨骼髋关节和膝关节构成了一个串并 联机构。该机构可以通过带动小腿末端按照人体 标准步态运动, 来精确模拟人体膝关节的复杂耦 合运动。

\section{2 基本原理}

图 2 为下肢外骨骼机构简化原理图, 由图 2 可 知, 参考坐标系 $O_{X O 1 Y}$ 固定在基座上, $O_{3}$ 点是末端 执行器。该机构包含三个直线电动机驱动器, 驱动 器 $D E, H O_{3}, B O_{3}$ 长度分别为 $L_{0}, L_{1}, L_{2}$ 。 $\theta_{1}$ 是人 体髋关节运动的角度, $\theta_{2}, \theta_{3}$ 分别是 $\mathrm{HO}_{3}, \mathrm{BO}_{3}$ 与 坚直方向所成的角度, $\varphi$ 是末端执行器转动的角度。

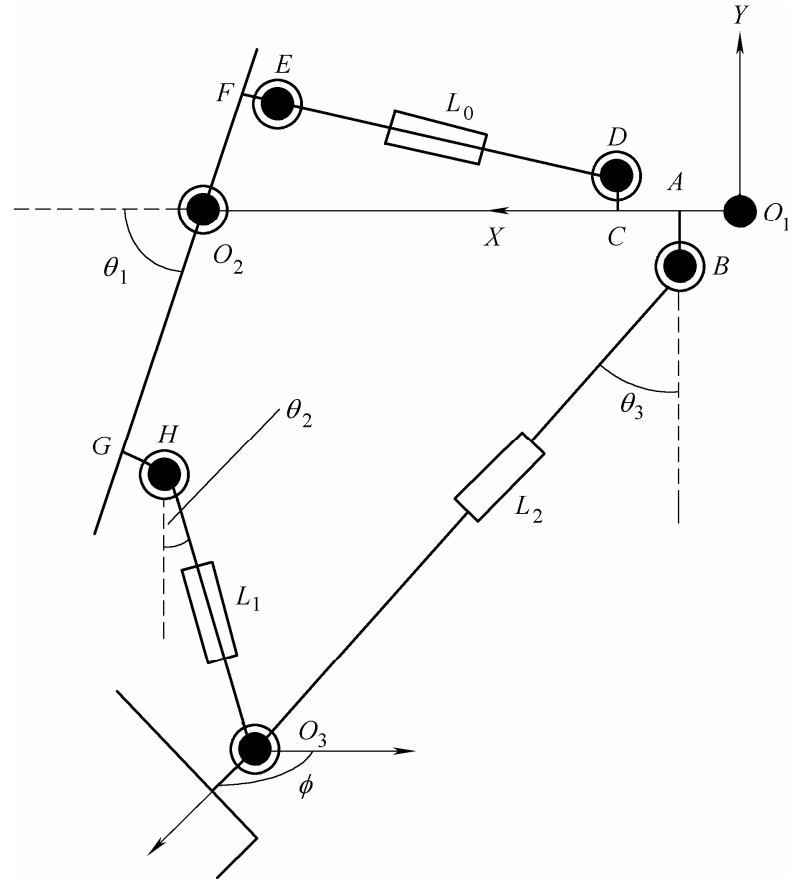

图 2 下肢外骨骼机构简化原理图

\section{3 自由度分析}

自由度计算公式 ${ }^{[16]}$ 可表示为

$$
F=d(n-g-1)+\sum_{i=1}^{g} f_{i}
$$

式中, $F$ 为机构的自由度数, $n$ 为机构的构件数, $g$
为运动副数, $f_{i}$ 为第 $i$ 个运动副的相对自由度, $d=6-\lambda, d$ 为机构的阶数, $\lambda$ 为机构中存在的公共 约束数。对于该机构, 机构的自由度为

$$
F=d(n-g-1)+\sum_{i=1}^{g} f_{i}=3 \times(8-9-1)+\sum_{1}^{9} 1=3
$$

基于以上分析可知，该机构有三个自由度，可 以实现沿平面 $X, Y$ 轴移动以及绕 $Z$ 轴的转动。

\section{4 位置正解}

机器人位置正解指在给定组成运动副相邻连 杆的相邻位置情况下, 确定机器人末端执行器的位 形。并联机器人的运动学正解问题可以通过令每个 分支运动链所确定的末端执行器的位置相等来 描述。

运用指数积公式对并联机构的位置正解进行 分析。基于指数积公式的正向运动学公式为 ${ }^{[14-15]}$

$$
g_{S T}(\boldsymbol{\theta})=\exp \left(\hat{\boldsymbol{\xi}}_{1} \theta_{1}\right) \cdots \exp \left(\hat{\boldsymbol{\xi}}_{n} \theta_{n}\right) g_{S T}(0)
$$

式中, $\theta_{i}$ 为机器人各关节的角度, $\hat{\xi}_{i}$ 与 $\boldsymbol{\xi}_{i}$ 的运算关 系为 $\boldsymbol{\xi}_{i}^{\wedge}=\hat{\boldsymbol{\xi}}_{i}, \boldsymbol{\xi}_{i}$ 为各连杆的运动旋量, $\exp \left(\hat{\xi}_{i} \theta_{i}\right)$ 为 相对应的旋转运动的运动旋量的指数积。 ${ }_{T}^{S} g(0)$ 表 示机器人位于初始位形惯性坐标系与工具坐标系间 的刚体变换。 $\{S\}$ 是惯性坐标系， $\{T\}$ 是与末端执行 器固联的工具坐标系。

对于每个关节都可以构造一个单位运动旋量 $\xi_{i}$, 这时除了第 $i$ 个关节之外其他关节均固定于初始 位形。

对于转动副

$$
\boldsymbol{\xi}_{i}=\left(\begin{array}{c}
\omega_{i} \\
\boldsymbol{r}_{i} \times \boldsymbol{\omega}_{i}
\end{array}\right)
$$

对于移动副

$$
\boldsymbol{\xi}_{i}=\left(\begin{array}{c}
0 \\
\boldsymbol{v}_{i}
\end{array}\right)
$$

式中, $\boldsymbol{\omega}_{i}$ 为运动轴线方向上的单位矢量, $\boldsymbol{r}_{i}$ 为轴线 上任意一点， $\boldsymbol{v}_{i}$ 是指移动方向的单位矢量。式(5)为 矩阵的指数形式, 式(5)中 $\boldsymbol{I}$ 为单位矩阵。 $\hat{\boldsymbol{\omega}}$ 是 $\boldsymbol{\omega}$ 的 反对称矩阵

$$
\exp (\theta \hat{\boldsymbol{\xi}})=\left(\begin{array}{cc}
\exp (\theta \hat{\boldsymbol{\omega}}) & (\boldsymbol{I}-\exp (\theta \hat{\boldsymbol{\omega}}))(\boldsymbol{\omega} \times \boldsymbol{v})+\theta \boldsymbol{\omega} \boldsymbol{\omega}^{\mathrm{T}} \boldsymbol{v} \\
0 & 1
\end{array}\right)
$$

$$
\exp (\theta \hat{\boldsymbol{\xi}})=\left(\begin{array}{cc}
\boldsymbol{I} & \theta \boldsymbol{v} \\
0 & 1
\end{array}\right) \quad \boldsymbol{\omega}=0
$$

针对第一条支链 $\mathrm{O}_{1} \mathrm{CDEFO} \mathrm{GHO}_{3}$, 建立图 3 所示的运动学数学模型。 


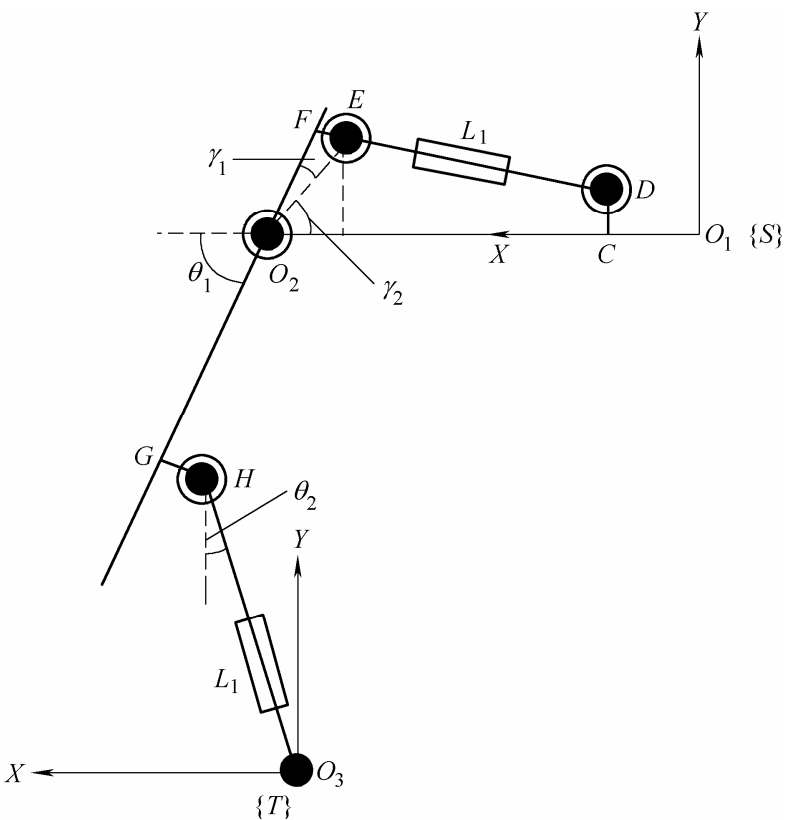

图 3 第一条支链数学模型

根据式(3) (5), 可以得到如下表达式

$$
\begin{array}{cc}
\boldsymbol{r}_{D}=\left(\begin{array}{c}
O_{1} C \\
C D \\
0
\end{array}\right) & \boldsymbol{r}_{E}=\left(\begin{array}{c}
X_{E} \\
Y_{E} \\
0
\end{array}\right) \\
\boldsymbol{r}_{O_{2}}=\left(\begin{array}{c}
O_{1} O_{2} \\
0 \\
0
\end{array}\right) & \boldsymbol{r}_{H}=\left(\begin{array}{c}
X_{H} \\
Y_{H} \\
0
\end{array}\right)
\end{array}
$$

式中, $O_{1} C, C D, O_{1} O_{2}$ 分别是固定连杆的距度, $X_{E}$, $Y_{E}$ 为 $E$ 点坐标, $X_{H}, Y_{H}$ 为 $H$ 点坐标

$$
\begin{aligned}
& E O_{2}=\sqrt{E F^{2}+F O_{2}^{2}} \quad \gamma_{1}=\arctan \left(\frac{E F}{F O_{2}}\right) \\
& \gamma_{2}=\theta_{1}-\gamma_{1} \\
& X_{E}=O_{1} O_{2}-E O_{2} \cos \gamma_{2} \quad Y_{E}=E O_{2} \sin \gamma_{2} \\
& X_{H}=O_{2} G \cos \theta_{1}-G H \sin \theta_{1} \\
& Y_{H}=-\left(O_{2} G \sin \theta_{1}+G H \cos \theta_{1}\right) \\
& \omega_{D}=\omega_{E}=\omega_{O_{2}}=\omega_{H}=\left(\begin{array}{l}
0 \\
0 \\
1
\end{array}\right) \\
& \boldsymbol{v}_{L_{0}}=\left(\begin{array}{l}
1 \\
0 \\
0
\end{array}\right) \quad \boldsymbol{v}_{L_{1}}=\left(\begin{array}{l}
0 \\
1 \\
0
\end{array}\right) \\
& \exp \left(\varphi_{1} \hat{\xi}_{1}\right)=\left(\begin{array}{cccc}
\cos \varphi_{1} & -\sin \varphi_{1} & 0 & C D \sin \varphi_{1}+O_{1} C\left(1-\cos \varphi_{1}\right) \\
\sin \varphi_{1} & \cos \varphi_{1} & 0 & -O_{1} C \sin \varphi_{1}+C D\left(1-\cos \varphi_{1}\right) \\
0 & 0 & 1 & 0 \\
0 & 0 & 0 & 1
\end{array}\right)
\end{aligned}
$$

$\exp \left(\varphi_{2} \hat{\boldsymbol{\xi}}_{2}\right)=\left(\begin{array}{cccc}\cos \varphi_{2} & -\sin \varphi_{2} & 0 & Y_{E} \sin \varphi_{1}+X_{E}\left(1-\cos \varphi_{1}\right) \\ \sin \varphi_{2} & \cos \varphi_{2} & 0 & -X_{E} \sin \varphi_{1}+Y_{E}\left(1-\cos \varphi_{1}\right) \\ 0 & 0 & 1 & 0 \\ 0 & 0 & 0 & 1\end{array}\right)$

$$
\exp \left(\theta_{1} \hat{\boldsymbol{\xi}}_{3}\right)=\left(\begin{array}{cccc}
\cos \theta_{1} & -\sin \theta_{1} & 0 & O_{1} O_{2}\left(1-\cos \theta_{1}\right) \\
\sin \theta_{1} & \cos \theta_{1} & 0 & -O_{1} O_{2} \sin \theta_{1} \\
0 & 0 & 1 & 0 \\
0 & 0 & 0 & 1
\end{array}\right)
$$

$\exp \left(\theta_{2} \hat{\boldsymbol{\xi}}_{4}\right)=\left(\begin{array}{cccc}\cos \theta_{2} & -\sin \theta_{2} & 0 & Y_{H} \sin \varphi_{1}+X_{H}\left(1-\cos \varphi_{1}\right) \\ \sin \theta_{2} & \cos \theta_{2} & 0 & -X_{H} \sin \varphi_{1}+Y_{H}\left(1-\cos \varphi_{1}\right) \\ 0 & 0 & 1 & 0 \\ 0 & 0 & 0 & 1\end{array}\right)$

$$
\exp \left(L_{0} \hat{\boldsymbol{\xi}}_{L_{0}}\right)=\left(\begin{array}{cccc}
1 & 0 & 0 & L_{0} \\
0 & 1 & 0 & 0 \\
0 & 0 & 1 & 0 \\
0 & 0 & 0 & 0
\end{array}\right) \exp \left(L_{1} \hat{\boldsymbol{\xi}}_{L_{1}}\right)=\left(\begin{array}{cccc}
1 & 0 & 0 & 0 \\
0 & 1 & 0 & L_{1} \\
0 & 0 & 1 & 0 \\
0 & 0 & 0 & 0
\end{array}\right)
$$

在刚体变换中, 均采用的是齐次坐标。当机器 人运动副角度为 0 时, 初始位形为

$$
g_{S T}(0)=\left(\begin{array}{cccc}
1 & 0 & 0 & O_{1} O_{2}+G H \\
0 & 1 & 0 & -\left(G H+L_{1}\right) \\
0 & 0 & 1 & 0 \\
0 & 0 & 0 & 1
\end{array}\right)
$$

式中, $G H$ 是固定连杆的距离。根据公式(2), 计算 出末端执行器 $O_{3}$ 点的位置

$$
\begin{gathered}
g_{S T}(\boldsymbol{\theta})=\exp \left(\varphi_{1} \hat{\boldsymbol{\xi}}_{1}\right) \exp \left(\varphi_{2} \hat{\boldsymbol{\xi}}_{2}\right) \exp \left(\theta_{1} \hat{\boldsymbol{\xi}}_{3}\right) \exp \left(\theta_{2} \hat{\boldsymbol{\xi}}_{4}\right) \cdots \times \\
\exp \left(L_{0} \hat{\boldsymbol{\xi}}_{L_{0}}\right) \exp \left(L_{1} \hat{\boldsymbol{\xi}}_{L_{1}}\right) g_{S T}(0) \\
\varphi_{1}+\varphi_{2}=\frac{\pi}{2}
\end{gathered}
$$

针对第二条支链 $\mathrm{O}_{1} A B O_{3}$, 建立如图 4 所示的 运动学模型。

当 $\theta_{3}=0$ 时, 则初始位形为始位形为

$$
g_{S T}(0)=\left(\begin{array}{cccc}
1 & 0 & 0 & 0 \\
0 & 1 & 0 & -\left(A B+L_{2}\right) \\
0 & 0 & 1 & 0 \\
0 & 0 & 0 & 1
\end{array}\right)
$$

构造转动关节和移动关节运动旋量

$$
\boldsymbol{r}_{1}=\left(\begin{array}{c}
O_{1} A \\
0 \\
0
\end{array}\right) \quad \boldsymbol{r}_{2}=\left(\begin{array}{c}
0 \\
-A B \\
0
\end{array}\right) \quad \boldsymbol{\omega}_{1}=\left(\begin{array}{l}
0 \\
0 \\
1
\end{array}\right) \quad \boldsymbol{v}_{1}=\left(\begin{array}{c}
0 \\
-1 \\
0
\end{array}\right)
$$




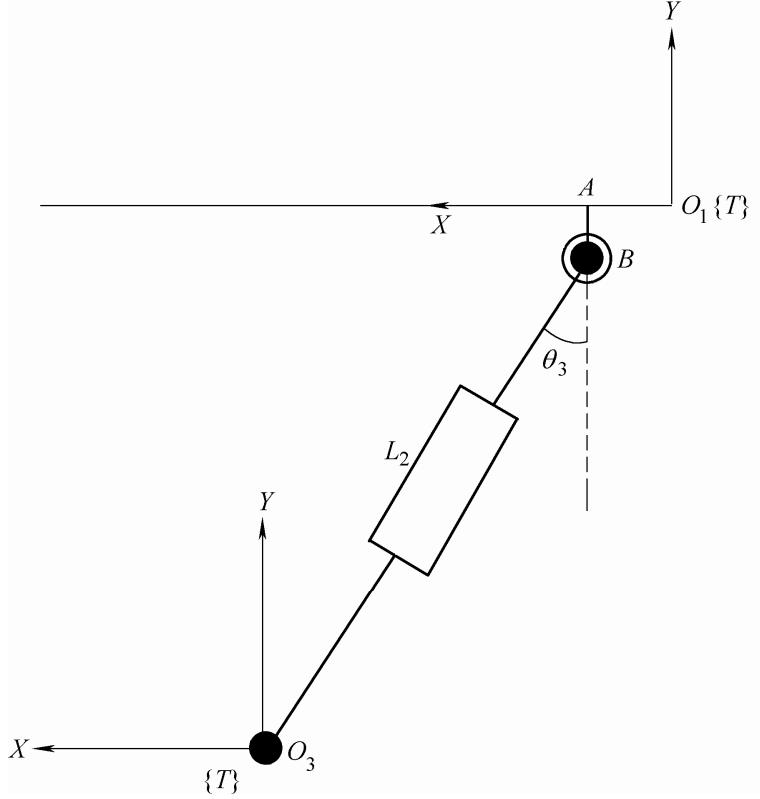

图 4 第二条支链数学模型

式(22)中 $O_{1} A, A B$ 是固定连杆的距离, 根据式(3) (6)得到如下表达式

$$
\begin{gathered}
\exp \left(\theta_{3} \hat{\xi}_{1}\right)=\left(\begin{array}{cccc}
\cos \theta_{3} & -\sin \theta_{3} & 0 & O_{1} A\left(1-\cos \theta_{3}\right) \\
\sin \theta_{3} & \cos \theta_{3} & 0 & -O_{1} A \sin \theta_{3} \\
0 & 0 & 1 & 0 \\
0 & 0 & 0 & 1
\end{array}\right) \\
\exp \left(L_{2} \hat{\xi}_{2}\right)=\left(\begin{array}{cccc}
1 & 0 & 0 & 0 \\
0 & 1 & 0 & -L_{2} \\
0 & 0 & 1 & 0 \\
0 & 0 & 0 & 1
\end{array}\right)
\end{gathered}
$$

根据式(2)可知

$$
\begin{aligned}
& g_{S T}(\boldsymbol{\theta})= \\
& \left(\begin{array}{cccc}
\cos \theta_{3} & -\sin \theta_{3} & 0 & \begin{array}{l}
\sin \theta_{3}\left(A B+L_{2}\right)+L_{2} \sin \theta_{3}- \\
O_{1} A\left(\cos \theta_{3}-1\right)
\end{array} \\
& & & -\cos \theta_{3}\left(A B+L_{2}\right)-O_{1} A \sin \theta_{3}- \\
\sin \theta_{3} & -\cos \theta_{3} & 0 & \begin{array}{l}
L_{2} \cos \theta_{3} \\
0
\end{array} \\
0 & 1 & 0 \\
0 & 0 & 0 & 1
\end{array}\right)
\end{aligned}
$$

并联机构的位置正解可以用指数坐标表示为

$$
\begin{gathered}
g_{S T}(\boldsymbol{\theta})=\exp \left(\theta_{1} \hat{\boldsymbol{\xi}}_{1}\right) \cdots \exp \left(\theta_{n} \hat{\boldsymbol{\xi}}_{\mathrm{n}}\right) g_{S T}(0)= \\
\exp \left(\varphi_{1} \hat{\boldsymbol{\xi}}_{1}\right) \cdots \exp \left(\varphi_{m} \hat{\boldsymbol{\xi}}_{m}\right) g_{S T}^{\prime}(0)
\end{gathered}
$$

式(26)中所有的参数都是相对于基础和工具坐标系 描述的。式(26)建立机器人关节转角之间的约束。
正是因为这些约束的存在，从而仅需要确定关节变 量的子集就能控制末端执行器的位置, 而其余关节 变量的取值必须满足式(26)。

由公式(26)可知, 该并联机构位置正解必须满 足如下表达式

$$
\begin{gathered}
g_{S T}(\boldsymbol{\theta})=\exp \left(\varphi_{1} \hat{\boldsymbol{\xi}}_{1}\right) \exp \left(\varphi_{2} \hat{\boldsymbol{\xi}}_{2}\right) \exp \left(\theta_{1} \hat{\xi}_{3}\right) \exp \left(\theta_{2} \hat{\boldsymbol{\xi}}_{4}\right) \cdots \times \\
\exp \left(L_{0} \hat{\boldsymbol{\xi}}_{L_{0}}\right) \exp \left(L_{1} \hat{\xi}_{L_{1}}\right) g_{S T}(0)= \\
\left(\begin{array}{cccc}
\cos \theta_{3} & -\sin \theta_{3} & 0 & \sin \theta_{3}\left(A B+L_{2}\right)+L_{2} \sin \theta_{3}-O_{1} A\left(\cos \theta_{3}-1\right) \\
\sin \theta_{3} & \cos \theta_{3} & 0 & -\cos \theta_{3}\left(A B+L_{2}\right)-O_{1} A \sin \theta_{3}-L_{2} \cos \theta_{3} \\
0 & 0 & 1 & 0 \\
0 & 0 & 0 & 1
\end{array}\right)
\end{gathered}
$$

\section{5 位置逆解}

机器人位置逆解就是给定末端执行器期望位 形的情况下, 求出满足该位形情况下的所有关节转 角。机器人的运动学逆解可能有很多解, 可能有唯 一解, 也可能无解。并联机器人的运动学逆解问题 可以通过对连接基座和末端执行机构的各开链机构 运动学逆解的处理来解决。PADEN 提出了将整个运 动学逆解的求解问题分解成若干个具有明确意义的 子问题 ${ }^{[16-17]}$, 并得到了广泛的应用。

对于 $n$ 自由度的开链机器人, 其位置正解为

$$
{ }_{T}^{S} g(\boldsymbol{\theta})=\exp \left(\theta_{1} \hat{\boldsymbol{\xi}}_{1}\right) \cdots \exp \left(\theta_{i} \hat{\boldsymbol{\xi}}_{i}\right) \cdots \exp \left(\theta_{n} \hat{\boldsymbol{\xi}}_{n}\right)_{T}^{S} g(0)
$$

式(28)左乘 ${ }_{T}^{S} g^{-1}(0)$, 可以写成

$$
{ }_{T}^{S} g(\boldsymbol{\theta})_{T}^{S} g^{-1}(0)=\exp \left(\theta_{1} \hat{\boldsymbol{\xi}}_{1}\right) \cdots \exp \left(\theta_{i} \hat{\boldsymbol{\xi}}_{i}\right) \cdots \exp \left(\theta_{n} \hat{\boldsymbol{\xi}}_{n}\right)
$$

$\hat{\boldsymbol{\xi}} \in \operatorname{se}(3)$ 和 ${ }_{T}^{S} g(\boldsymbol{\theta}) \in S E(3)$ 均是已知。 $\theta_{i}$ 是待求。

已知末端执行器的坐标为 $O_{3}(X, Y)$, 根据 PADEN 子问题 $2^{[17]}$, 求出关节角满足以下表达式。

$$
X=O_{1} A+L_{2} \sin \theta_{3} \quad Y=-\left(A B+L_{2} \cos \theta_{3}\right)
$$

$O_{2} G \cos \theta_{1}-G H \sin \theta_{1}-L_{1} \sin \theta_{2}=O_{1} A+L_{2} \sin \theta_{3}$

$O_{2} G \sin \theta_{1}-G H \cos \theta_{1}-L_{1} \cos \theta_{2}=A B+L_{2} \cos \theta_{3}$

\section{6 机构的雅可比矩阵及其奇异性}

机构各支链的伸缩速度为 $\dot{\boldsymbol{L}}$, 末端执行的广义 速度为 $\dot{\boldsymbol{X}}$, 其中 $\dot{\boldsymbol{L}}=\left(\dot{L}_{1} \dot{L}_{2}\right)^{\mathrm{T}}, \dot{\boldsymbol{X}}=\left(\begin{array}{ll}\dot{X} & \dot{Y}\end{array}\right)^{\mathrm{T}}$ 。机构 的输入输出速度关系满足 $\dot{\boldsymbol{X}}=\boldsymbol{J} \dot{\boldsymbol{L}}$, 其中 $\boldsymbol{J}=\boldsymbol{J}_{2}^{-1} \boldsymbol{J}_{1}, \boldsymbol{J}_{1}$ 和 $\boldsymbol{J}_{2}$ 分别表示机构的逆, 正雅可比 矩阵 ${ }^{[18]}$ 。可表示为

$$
\boldsymbol{J}_{1}=\left(\begin{array}{cc}
\dot{L}_{1} & 0 \\
0 & \dot{L}_{2}
\end{array}\right)
$$


$\boldsymbol{J}_{2}=\left(\begin{array}{cc}X-O_{2} G \cos \theta_{1}+G H \sin \theta_{1} & Y+O_{2} G \sin \theta_{1}+G H \cos \theta_{1} \\ X-O_{1} A & Y+A B\end{array}\right)$

$\boldsymbol{J}$ 为机构位姿速度对各支链输入速度的一阶影 响系数矩阵，即通常所说的 Jacobian 矩阵。

可以看出, 机构的逆雅可比矩阵 $\boldsymbol{J}_{1}$ 的行列式不 可能为 0 , 故以正雅可比矩阵 $\boldsymbol{J}_{2}$ 为基础, 对机构的 奇异位形进行分析。令 $\operatorname{det}\left(\boldsymbol{J}_{2}\right)=0$, 整理得

$$
\begin{gathered}
a_{1}=-O_{2} G \cos \theta_{1}-G H \sin \theta_{1} \quad a_{3}=O_{1} A \\
\left(X-a_{1}\right)\left(Y-a_{4}\right)-\left(Y-a_{2}\right)\left(X-a_{3}\right)=0 \\
a_{2}=-O_{2} G \sin \theta_{1}-G H \cos \theta_{1} a_{4}=-A B
\end{gathered}
$$

由式(36)可知, 当 $a_{1}=a_{3}, a_{2}=a_{4}$ 时, 即此时 $B$ 点与 $H$ 点重合, 在实际中, 由于关节角度和运动副转角的 限制, 这种奇异位形是可以避免的。

\section{2 工作空间分析}

\section{1 定姿态工作空间}

机器人工作空间的大小代表了机器人的活动 范围, 它是衡量机器人工作能力的一个重要运动学 指标。并联机构的工作空间是指并联机构操作器的 工作区域 ${ }^{[19]}$ 。选取并联机构末端执行器 $O_{3}$ 作为研 究工作空间的参考点, 并联工作空间的求解就是在 满足杆长尺寸和关节变量范围的条件下, $O_{3}$ 点所能 达到的空间集合，如图 5 所示。

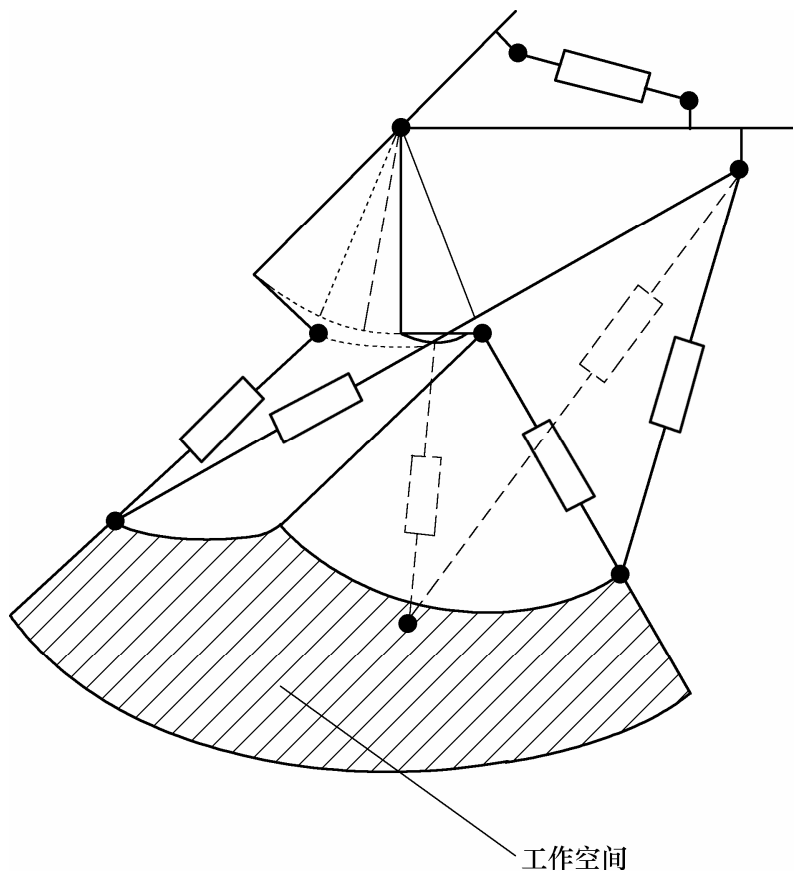

图 $5 O_{3}$ 点所能达到的空间集合

\section{2 工作空间的主要约束条件}

杆长约束

$$
L_{\min } \leqslant L_{i} \leqslant L_{\max }
$$

式中, $L_{\min }, L_{\max }$ 分别表示直线电动推杆杆长的最小 值和最大值。

关节角约束。关节角度约束是由于人体髋关 节, 膝关节以及踝关节正常行走时产生。关节角度 的约束条件为

$$
\theta_{1 \min } \leqslant \theta_{1} \leqslant \theta_{1 \max }
$$

式中, $\theta_{1 \mathrm{~min}}, \theta_{1 \max }$ 是对应关节运动角度的最小值和 最大值。

转动副的转角限制。转动副在连接机构连杆的 同时，也要求转角不能随意转动，必须符合设计的 要求。这里定义连杆与坚直方向的夹角为 $\theta_{i}(i=2$, $3), \theta_{i}$ 应满足的条件是

$$
\theta_{i \min } \leqslant \theta_{i} \leqslant \theta_{i \max }
$$

式中, $\theta_{i \min }, \theta_{i \max }$ 是对应转动副的转角角度的最小 值和最大值。

\section{3 工作空间面积建模}

并联机构工作空间的面积随着结构参数的变 化而变化, 这些机构的主要参数有并联杆长度 $L_{\mathrm{i}}(i=0$, $1,2)$ 、关节角度 $\theta_{1}$ 、转动副转角 $\theta_{2}, \theta_{3}$ 、并联杆安装 位置 $a, b \circ a$ 为 $A C$ 之间的距离, $b$ 为 $O_{2} G$ 之间的距 离。工作空间的面积可看成是机构参数的函数。

$$
\begin{gathered}
L_{2}{ }^{2}=\left(O_{2} G \cos \theta_{1}-G H \sin \theta_{1}-L_{1} \sin \theta_{2}-O_{1} A\right)^{2}+ \\
\left(O_{2} G \sin \theta_{1}-G H \cos \theta_{1}-L_{1} \cos \theta_{2}-A B\right)^{2} \\
S=F\left(L_{i}, \theta_{i}, a, b\right)=\frac{\pi}{9}\left[\left(L_{2}+63\right)^{2}-L_{2}{ }^{2}\right]
\end{gathered}
$$

工作空间的面积模型为多元函数, 此函数可以由数 值法求得的空间的面积。

在实际的问题中, 希望所设计的结构参数尽可 能地使整个机构具有较大的空间，并希望整体的驱 动性能最好。因此选取机构参数优化的目标函数

$$
F(X, Y)=\max S
$$

机构参数的约束条件如表 1、表 2 所示。

表 1 电动机长度表 $(\mathrm{mm})$

\begin{tabular}{ccc}
\hline 电动机长度 & 最小值 & 最大值 \\
\hline$L_{0}$ & 377 & 440 \\
$L_{1}$ & 370 & 450 \\
$L_{2}$ & 410 & 480 \\
\hline
\end{tabular}

表 2 关节运动角度表 $\left(^{\circ}\right)$

\begin{tabular}{ccc}
\hline 关节运动角度 & 最小值 & 最大值 \\
\hline$\theta_{1}$ & 0 & 80 \\
$\theta_{2}$ & -30 & 30 \\
$\theta_{3}$ & 20 & 60 \\
\hline
\end{tabular}

目标函数 $F(X)=\max S$; 优化参数 $a, b$; 已 知参数 $A B=C D=75 \mathrm{~mm}, A C=a, E F=38 \mathrm{~mm}$, $O_{1} O_{2}=474 \mathrm{~mm}, O_{1} A=35 \mathrm{~mm}, G O_{2}=b, G H=40 \mathrm{~mm}$, $\mathrm{FO}_{2}=114 \mathrm{~mm}$; 约束条件如下 


$$
\begin{gathered}
0^{\circ} \leqslant \theta_{1} \leqslant 80^{\circ} \quad-30^{\circ} \leqslant \theta_{2} \leqslant 30^{\circ} \quad-20^{\circ} \leqslant \theta_{3} \leqslant 60^{\circ} \\
0<a \leqslant 200 \quad 0<b<400 \\
L_{0}{ }^{2}=\left(X_{E}-O_{1} C\right)^{2}+\left(Y_{E}-C D\right)^{2} \\
L_{2}{ }^{2}=\left(O_{2} G \cos \theta_{1}-G H \sin \theta_{1}-L_{1} \sin \theta_{2}-O_{1} A\right)^{2}+ \\
\left(O_{2} G \sin \theta_{1}-G H \cos \theta_{1}-L_{1} \cos \theta_{2}-A B\right)^{2}
\end{gathered}
$$

其中 $A C=a, O_{2} G=b$, 由式(41)、(42)得到具体 参数, 应用算法进行优化。通过 Matlab 软件计算出 工作空间最大面积为 $S_{\max }=8765.34 \mathrm{~mm}^{2}$, 此时 $a=180.17 \mathrm{~mm}, b=345.78 \mathrm{~mm}$ 。此时的工作空间面积 最大, 运动范围更加接近人体下肢运动范围。

\section{4 分析与讨论}

机器人运动学是机器人路径规划基础, 通过运 用指数积公式求解并联机构的位置正反解, 来实现 机器人末端以特定的姿态沿给定的路径运动。设定 机器人末端的起点坐标为 $(-500,800)$, 终点坐标为 $(0$, -400), 机器人末端从起点到终点进行直线运动。机 器人末端的初始速度, 初始加速度设为已知。通过 正逆运动学计算, 绘制出关节角度以及各电动机长 度的变化值, 如图 6 12 所示。

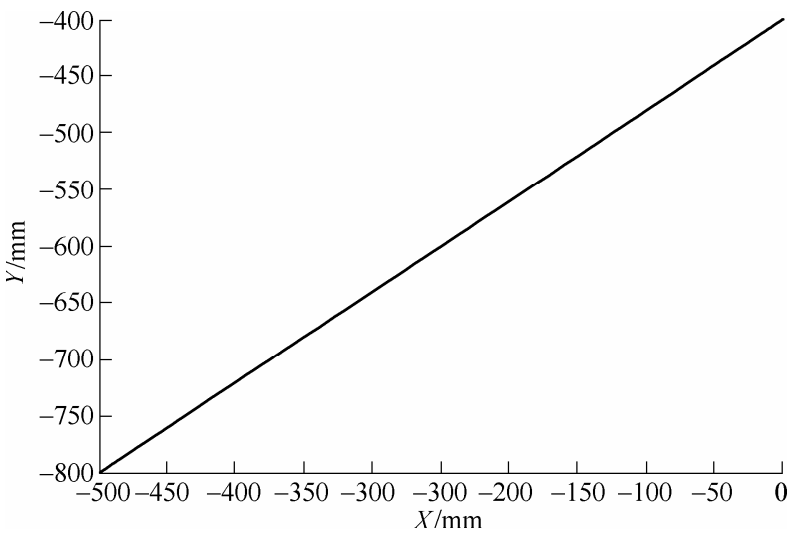

图 6 机器人末端运动轨迹

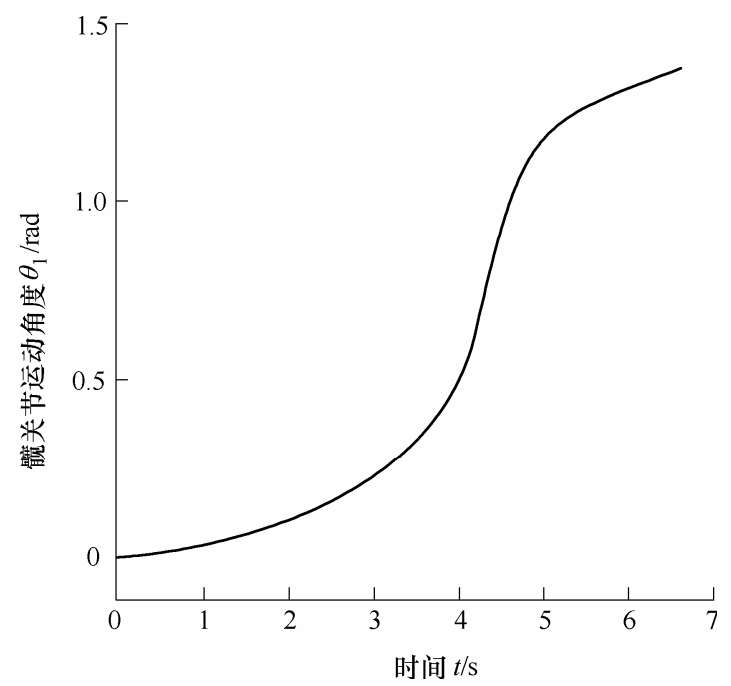

图 7 髋关节运动角度 $\theta_{1}$ 变化值

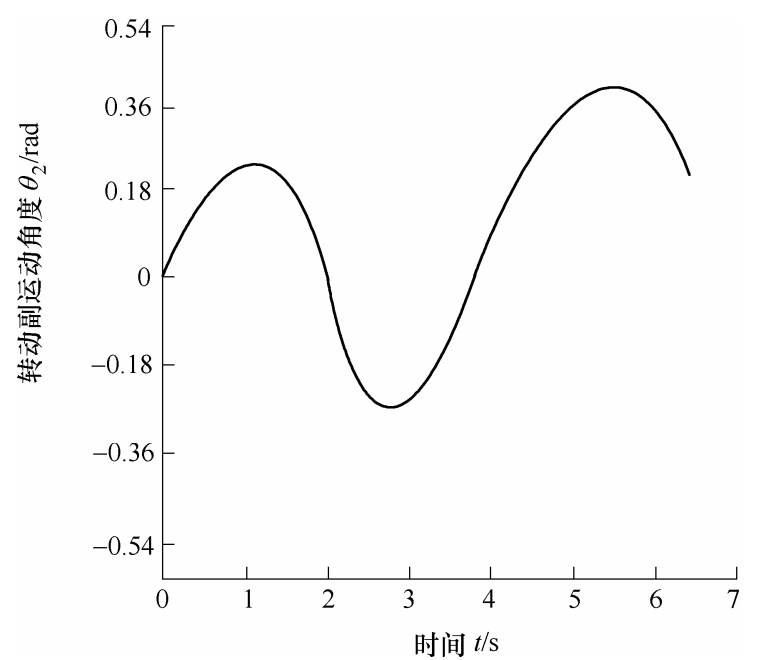

图 8 膝关节运动角度 $\theta_{2}$ 变化值

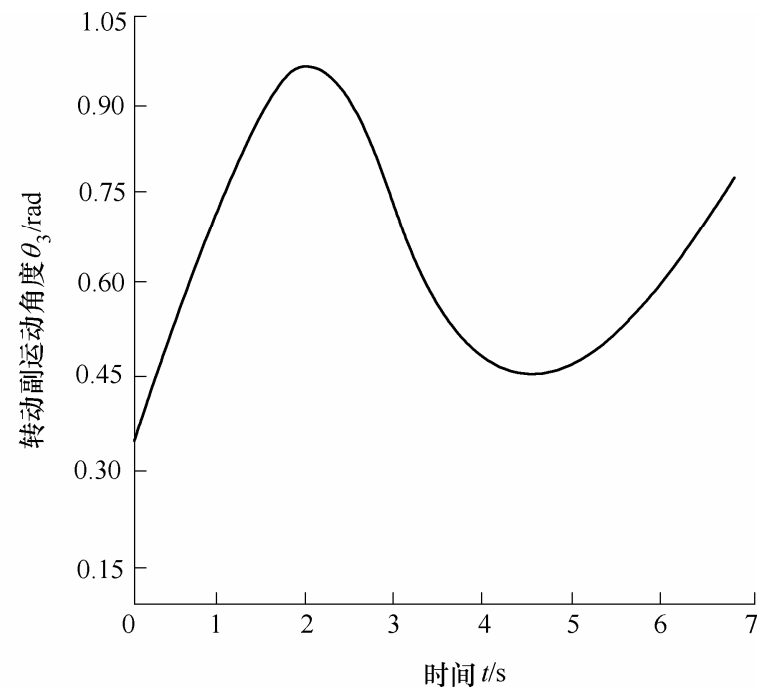

图 9 膝关节运动角度 $\theta_{3}$ 变化值

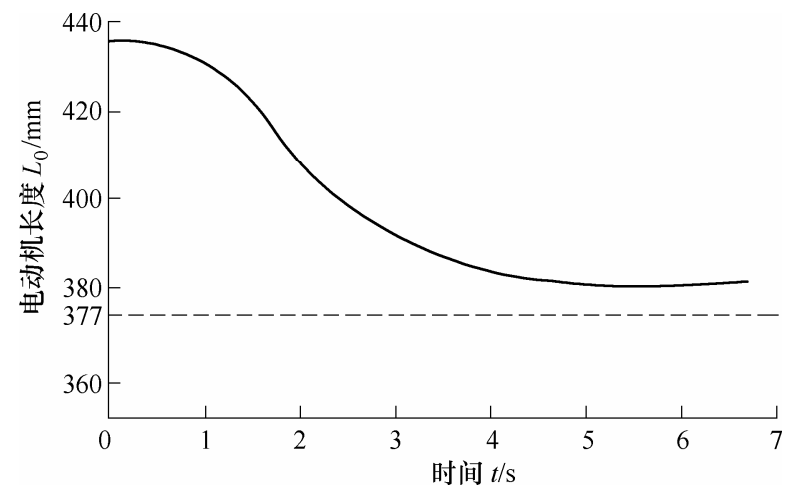

图 10 电动机长度 $L_{0}$ 变化值

通过轨迹规划来对机器人运动学位置正逆解 进行验证, 试验结果表明电动机推杆长度, 人体关 节角度以及运动副转角都在约束范围内, 符合设计 要求。针对设计的下肢外骨骼机构, 运用指数积公 式进行正逆运动学分析, 使机器人末端执行器可以 按照设定的轨迹进行运动, 对下肢功能障碍患者进 行康复训练。 


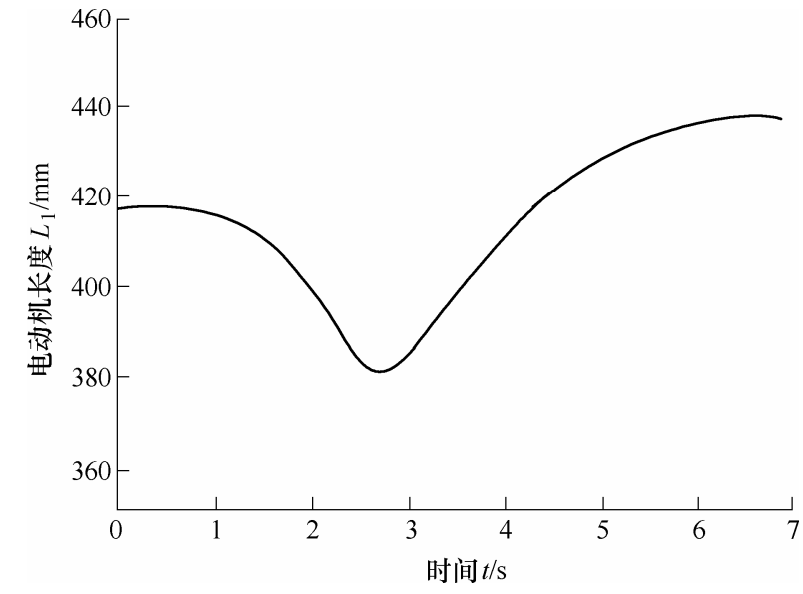

图 11 电动机长度 $L_{1}$ 变化值

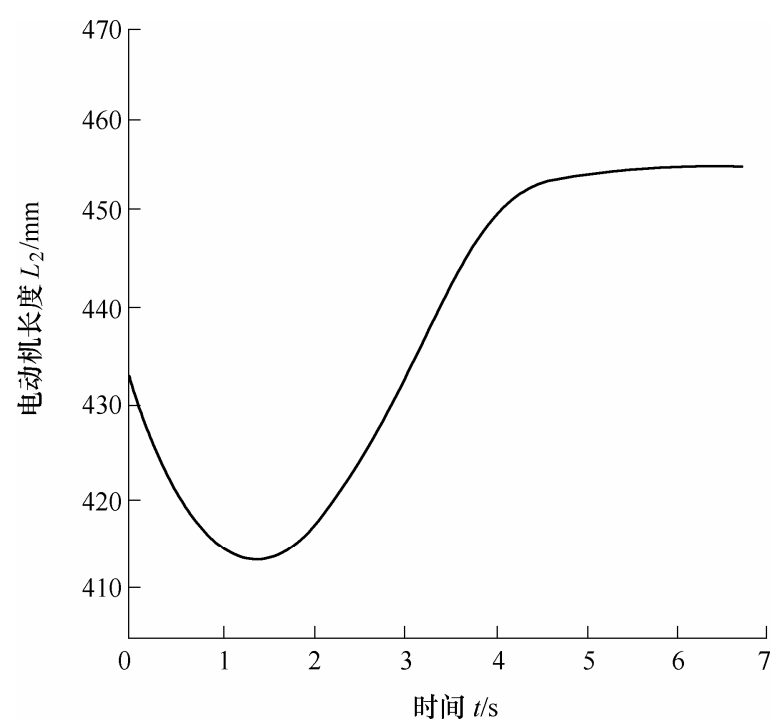

图 12 电动机长度 $L_{2}$ 变化值

\section{3 结论}

(1) 对平面三自由度并联机构进行运动学分 析, 运用指数积公式求得了该机构的位置正反解, 对机构的雅可比矩阵和运动奇异性进行了分析, 为 新型的并联机构的研究提供了理论基础。

(2) 给出约束条件的基础上, 以提高运动学性 能为指标, 以工作空间最大为目标函数, 优化结构 参数。

（3）借助 Matlab 强大的数学分析能力编程能 力, 对平面并联机构的工作空间进行了仿真, 结果 显示该工作空间结构紧凑, 没有空洞, 因此该机构 具有良好的工作能力。

（4）本文的研究结果为平面并联机构进一步的 动力学分析、轨迹规划、优化设计以及扩展到三维 的并联机构的研究奠定了基础。

\section{参 考 文 献}

[1] 黄真. 并联机器人机构学理论及控制 [M]. 北京: 机械 工业出版社, 1997

HUANG Zhen. Theory and control of parallel robot mechanism[M]. Beijing: China Machine Press, 1997.

[2] RIENER R, MAIER I C. Locomotor training in subjects with sensori-motor deficits: An overview of the robotic gait orthosis lokomat[J]. Journal of Healthcare Engineering, 2010, 1(2): 197-216.

[3] ZANOTTO, DAMIANO, STEGALl P, et al. Adaptive assist-as-needed controller to improve gait symmetry in robot-assisted gait training[C]// The IEEE International Conference on Robotics and Automation, May 31-June 7, 2014, Hong Kong, China. Hong Kong: ICRA, 2014: 724-729.

[4] LOW K H. Robot-assisted gait rehabilitation: From exoskeletons to gait systems $[\mathrm{C}] / /$ Defense Science Research Conference and Expo, August3-5, 2011, Singapore. Singapore: IEEE, 2011: 1-10.

[5] 刘慧林, 张同庄, 丁洪生. 3-RPR 平面并联结构正解的 吴方法 [J]. 北京理工大学学报, 2000, 20(5): 565-569. LIU Huilin, ZHANG Tongzhuang, DING Hongsheng. Forward solution of the 3-RPR planar mechanism with Wu's method[J]. Journal of Beijing Institute of Technology, 2000, 20(5): 565-569.

[6] 罗佑新. 3-RPR 平面并联结构正解的混沌方法 [J]. 机械 科学与技术，2004(8): 916-918.

LUO Youxin. Forward solutions of the 3-RPR planar parallel mechanism with chaos solution method[J]. Mechanical Science and Technology, 2004(8): 916-918.

[7] 高征, 高峰. 新型并联机器人的奇异位形分析[J]. 机械 工程学报，2008，44(1): 133-138.

GAO Zheng, GAO Feng. Singularity of Loci analysis of a new parallel manipulator[J]. Chinese Journal of Mechanical Engineering, 2008， 44(1): 133-138.

[8] 程世利, 吴洪涛. 平面平台型 Stewart 并联机构的奇异 位形分析[J]. 机械工程学报, 2011，47(9): 1-7.

CHENG Shili, WU Hongtao. Singularity analysis of Stewart parallel mechanism with planar platform[J]. Journal of Mechanical Engineering, 2001, 47(9): 1-7.

[9] LI Haidong, GOSSLIN C M, RICHARD M J. Determination of maximal singularity-free zones in the workspace of the planar three-degree-of-freedom parallel mechanisms[J]. Mechanism and Machine Theory, 2006, 41: 1157-1167.

[10] 赵新华, 彭商贤. 并联机器人奇异位形研究 [J]. 天津理 
工学院学报, 2000, 36(5): 35-37.

ZHAO Xinhua, PENG Shangxian. Singularity analysis of parallel robot[J]. Journal of Tianjin University of Technology, 2000, 36(5): 35-37.

[11] GOSSLIN C M, JEAN M. Determination of the workspace of planar manipulators with joint limits[J]. Robotics and Autonomous Systems, 1996, 17(30): 129-138.

[12] 肖红军. 3-RPR 平面并联机器人工作空间研究 $[J]$. 电子 科技，2010，23(10): 114-117.

Xiao Hongjun. Study of workspace of the planar 3-RPR parallel manipulator[J]. Electronic Sci\&Tech., 2010, 23(10): 114-117.

[13] BOUDREAU R, GOSSELIN C. The synthesis of planar parallel manipulators with a genetic algorithm[J]. ASME J. Mech. Des., 1999, 121(4): 533-973.

[14] CHEN Xuesheng, CHEN Zaili, XIE Tiao. The synthesis of a Stewart platform for a specific workspace with a genetic algorithm[J]. High Technology Letters, 2004(3): 66-69.

[15] 马晓丽, 马履中, 周兆忠, 等. 3T-1R 并联平台的工作 空间分析与设计 $[\mathrm{J}]$. 中国机械工程, 2006, 17(18): 1938-1943.

MA Xiaoli, MA Lüzhong, ZHOU Zhaozhong, et al. Workspace analysis and optimal design of a 3T-1R parallel kinematics platform[J]. Chinese Journal of Mechanical Engineering, 2006, 17(18): 1938-1943.

[16] 于靖军, 刘辛军, 丁希仑, 等. 机器人机构学的数学基
础[M]. 北京: 机械工业出版社, 2009.

YU Jingjun, LIU Xinjun, DING Xilun, et al. Mathematic foundation of mechanisms and robotics[M]. Beijing: China Machine Press, 2009.

[17] 莫雷, 李泽湘, 萨思特里. 机器人操作的数学导论 $[\mathrm{M}]$. 北京：机械工业出版社，1998.

MURRAY R, LI Z X, SASTRY S. A mathematical introduction to robotic manipulation[M]. Beijing: China Machine Press, 1998.

[18] 何景峰, 李保平, 杨宏斌, 等. Gough-Stewart 机构的奇 异性及其 ADAMS 仿真验证 $[\mathrm{J}]$. 机床与液压, 2010, 38(5): 104-107.

HE Jingfeng. LI Baoping, YANG Hongbin, et al. Singularity of Gough-Stewart platform with ADAMS validation[J]. Machine Tools \& Hydraulics, 2010, 38(5): 104-107.

[19] CLEARY K, ARAI T. A prototype parallel manipulators: Kinematics, construction, software, workspace results, and singularity analysis[C]// International Conference on Robotics an Automation, April 1991, Sacramento, California, USA. USA: IEEE, 1991: 566-571.

作者简介: 陈伟海(通信作者), 男, 1955 年出生, 博士, 教授, 博士研 究生导师。主要研究方向为仿生机器人设计与控制、柔顺机构设计与控 制, 高精密运动机械设计与控制等。

E-mail: whchen@buaa.edu.cn

徐颖俊, 男, 1991 年出生。主要研究方向为仿生机器人设计与控制, 机 器人的智能检测和智能运动控制。

E-mail: xuyingjun2012@163.com 